\title{
Annexin A3 and cancer (Review)
}

\author{
CHAO LIU* ${ }^{*}$ NANNAN LI*, GUIJIAN LIU and XUE FENG \\ Clinical Laboratory, Guang'anmen Hospital, China Academy of \\ Chinese Medical Sciences, Beijing 100053, P.R. China
}

Received August 5, 2021; Accepted September 29, 2021

DOI: 10.3892/ol.2021.13095

\begin{abstract}
Annexin A3 (ANXA3), an annexin family member, contains $36 \mathrm{kDa}$ and $33 \mathrm{kDa}$ isoforms. Similar to other annexin members, ANXA3 plays an important role in the development of human diseases. Recent studies have reported that abnormal ANXA3 expression is closely associated with the development, progression, metastasis, drug resistance and prognosis of several malignant tumours, such as breast cancer, lung cancer and hepatocellular carcinoma. ANXA3 exerts its role by regulating cell proliferation, migration and apoptosis via the phosphatidylinositol-3 kinase/Akt, nuclear factor- $\kappa \mathrm{B}(\mathrm{NF}-\kappa \mathrm{B})$, c-JUN N-terminal kinase, extracellular signal-regulated kinase and hypoxia-inducible factor-1 signalling pathways. ANXA3 may act as a novel target for the early diagnosis and treatment of tumours. The present review summarises the recent progress in the role of ANXA3 and its regulatory pathways in tumours.
\end{abstract}

\section{Contents}

1. Introduction

2. ANXA3 and tumour

3. Pathways by which ANXA3 exerts its role

4. Conclusion and prospects

\section{Introduction}

Annexins. Annexins are a well-known multigene family, which bind to phospholipids in a $\mathrm{Ca}^{2+}$-dependent manner (1). They are divided into five groups (groups A, B, C, D and E), and are expressed in mammals, invertebrate, Mycetozoa and fungi, plants and Protista, respectively (1-3). Group A annexins have

Correspondence to: Mrs. Xue Feng or Dr Guijian Liu, Clinical Laboratory, Guang'anmen Hospital, China Academy of Chinese Medical Sciences, 5 Bei Xiange Road, Beijing 100053, P.R. China

E-mail: fengxueimmunology@126.com

E-mail: liuguijian@163.com

${ }^{*}$ Contributed equally

Key words: Annexin A3, cancer, role, pathway been extensively studied due to their association with the development of human diseases. This group contains 12 members (annexins A1-A11 and annexin A13). The structure of annexins is similar; they are composed of a highly conserved C-terminus and a variable $\mathrm{N}$-terminus. The $\mathrm{C}$-terminal protein core contains four annexin repeats, each composed of 69-80 amino acid residues (4). The N-terminal domain is highly variable, in which the diversity of the amino acid sequence and length result in the difference of functions among all annexin family members $(4,5)$. Recent studies have reported that annexins play an important role in tumour development and progression, cell proliferation and apoptosis, invasion and metastasis, drug resistance and angiogenesis (6,7).

Annexin A3 (ANXA3), a memberof the annexinfamily, is also known as lipocortin III or placental anticoagulant protein III (3). ANXA3 is considered a new angiogenesis factor $(8,9)$, which participates in the liver regeneration process $(10,11)$. ANXA3 is also closely associated with the development of human diseases, such as cancer and inflammation $(3,12)$.

Structure of ANXA3. ANXA3 belongs to the annexin family, whose encoding gene is in human chromosome 4q13-q22 (12). Previous studies have demonstrated that the molecular structures of ANXA3 and ANXA5 are highly homologous $(13,14)$. The $\mathrm{Ca}^{2+}$ binding sites of both annexins lie on the convex face of the conversed domains, each of which contain helices A and B (13). The crystal structure of ANXA3 resembles that of ANXA8, which owns a complete and middle-length N-terminal region (14). This is because both annexins are found in promyelocytic cells undergoing the differentiation process (14). The C-terminal region of ANXA3 consists of four conserved domains (domains I-IV), forming a circular queue; each of them is composed of five $\alpha$-helices (helices A-E). The N-terminal region comprises 20 amino acid residues that can facilitate the modulation of phospholipid membrane binding and ion permeation via ANXA3. ANXA3 contains two special tryptophan residues, one situated in the N-terminal segment (W5), while the other is at the extremity of IIIA-IIIB loops (W190) (15-17). Both tryptophans can influence the interactions between ANXA3 and phospholipid membrane, $\mathrm{Ca}^{2+}(15-17)$.

Two isoforms of ANXA3. Increasing evidence suggest that the ANXA3 protein has two forms, one has a molecular weight of $33 \mathrm{kDa}$, while the other has a molecular weight of $36 \mathrm{kDa}$ (12). Previous studies have reported that most tumours only express the $36 \mathrm{kDa}$ form of the ANXA3 protein, while some cells 
simultaneously express both forms of the ANXA3 protein or just express either of the two forms ( $33 \mathrm{kDa}$ or $36 \mathrm{kDa}$ ), such as HL-60 myeloid cells, rat brain cells, prostate cancer cells, the retinal and choroidal endothelial cells (18-22). Le Cabec et al detected the expression of both forms of the ANXA3 protein in the human leukaemic myeloblast HL-60 cells via western blot analysis (18). The results indicated that the $33 \mathrm{kDa}$ and $36 \mathrm{kDa}$ isoforms of ANXA3 protein were synchronously present in undifferentiated HL-60 cells. When HL-60 cells were differentiated along the neutrophilic pathway or monocytic pathway, the $33 \mathrm{kDa}$ form of the ANXA3 protein was mainly expressed in blood neutrophils, while the $36 \mathrm{kDa}$ form of the ANXA3 protein was expressed in monocytes. Junker et al (20) determined that rat brain cells express the $33 \mathrm{kDa}$ and $36 \mathrm{kDa}$ isoforms of the ANXA3 protein. Further study demonstrated that the expression of the $33 \mathrm{kDa}$ isoform of ANXA3 is significantly upregulated in the rat brain following the occurrence of cerebral ischaemia (20). Using two-dimensional electrophoresis combined with western blotting, Bianchi et al (23) detected ANXA3 protein expression in primary cell cultures, which were isolated from 20 matched human renal cortical and renal cell carcinoma (RCC) tissues. The results demonstrated that the $33 \mathrm{kDa}$ and $36 \mathrm{kDa}$ isoforms of the ANXA3 protein were both expressed. Compared with renal cortical cultures, expression of the ANXA3 $33 \mathrm{kDa}$ form in RCC cultures significantly increased, whereas expression of the $36 \mathrm{kDa}$ form of the ANXA3 protein and total ANXA3 protein significantly decreased. Furthermore, tissue microarray analysis revealed that ANXA3 protein expression is downregulated in RCC tissues compared with normal renal cortical tissues.

Increasing evidence suggest that generation of the $33 \mathrm{kDa}$ and $36 \mathrm{kDa}$ isoforms of the ANXA3 protein has no association with post-translational modifications, such as phosphorylation and N-glycosylation (18). The mRNAs encoding ANXA3, which result from the alternative splicing of exon III, produce two ANXA3 transcripts, including the $36 \mathrm{kDa}$ isoform of the ANXA3 protein of a full-length protein and the $33 \mathrm{kDa}$ isoform of the ANXA3 protein, lacking the first 39 amino acid residues of the N-terminal region $(18,21,23)$ (Fig. 1). The generation model of the two ANXA3 transcripts is similar to that of the remaining members of the annexin family (2).

\section{ANXA3 and tumour}

ANXA3 and the development and progression of tumour. It has been reported that abnormal ANXA3 protein expression is closely associated with the development and progression of tumour. Overexpression of ANXA3 promotes the development and progression of tumours, such as upper tract urothelial cancer (UTUC), gastric carcinoma, hepatocellular carcinoma (HCC), breast cancer and osteosarcoma, whereas ANXA3 knockdown suppresses the tumorigenesis of prostate carcinoma (24-44) (Table I).

ANXA3 and the development and progression of upper tract urothelial cancer. Lu et al (24) investigated ANXA3 expression in urine samples and tissues, which were collected from 13 patients with UTUC and 20 healthy individuals, using non-fixed volume stepwise weak anion exchange chromatography coupled with two-dimensional electrophoresis assay. The results revealed 55 differential expression proteins in urine samples between patients with UTUC and healthy controls. High ANXA3 expression in patients with UTUC was further detected via western blot analysis. Taken together, these findings suggest that high ANXA3 expression is strongly associated with tumorigenesis of UTUC, and thus ANXA3 may act as a potential biomarker for diagnosing UTUC.

ANXA3 and the development and progression of gastric cancer. By performing quantitative PCR and western blot analyses, Yu et al (25) reported that ANXA3 expression is upregulated in gastric cancer tissues compared with adjacent normal tissues at both the protein and mRNA levels. The protein and mRNA expression levels of ANXA3 decreased following transfection with small interfering (si)RNA. In addition, ANXA3 knockdown suppressed the cell proliferation, migration and invasion of gastric cancer. Collectively, these findings suggest that ANXA3 may participate in the tumorigenesis of gastric cancer by acting as an oncogene. Researchers evaluated the expression intensity of ANXA3 in 80 gastric cancer tissues and investigated its association with prognosis, proliferation and apoptosis of gastric cancer by tissue microarray combined with immunohistochemistry (26). The results demonstrated that ANXA3 expression was higher in gastric cancer compared with the controls, which was positively associated with tumour volume and TNM stage.

$\mathrm{Ki}-34$ is a well-known proliferating cell nuclear antigen, and its function is strongly associated with mitosis (27). The proliferation index of $\mathrm{Ki}-34$ is frequently used to estimate the malignant proliferation of tumour cells in the clinic setting (27). Bcl-2 is an important indicator of the apoptotic signalling pathway, and is an antiapoptotic member of the $\mathrm{Bcl}-2$ protein family (28). Upregulated ANXA3 expression causes alterations in the expression levels of $\mathrm{Ki}-34$ and $\mathrm{Bcl}-2$, which significantly decreases the overall survival of patients with gastric cancer (26). Upregulated ANXA3 expression was associated with a poor prognosis among 183 patients with gastric cancer (29). Inhibition of ANXA3 suppresses tumorigenicity in vivo and suppresses the proliferation, clone formation, migration and invasion of gastric cancer cells (29). Taken together, these findings suggest that ANXA3 may serve as an independent marker of poor prognosis of gastric cancer. Recently, it has been demonstrated that ANXA3 expression in the Ochratoxin A (OTA) induces the malignant transformation of human gastric epithelium cells (GES-1) compared with normal GES-1 cells (30). The proliferative, migratory and invasive abilities of OTA-GES-1T cells weaken following ANXA3 knockdown (30). Overexpression of ANXA3 promotes the carcinogenesis of gastric cancer by regulating the cell proliferation and apoptosis of gastric cancer. However, further studies are required to determine the molecular mechanisms underlying the contribution of ANXA3 to the carcinogenesis of gastric cancer.

ANXA3 and the development and progression of HCC. ANXA3 expression was detected in 20 pairs of HCC tumour tissues and the corresponding normal tissues via immunohistochemical staining (31). The results demonstrated that ANXA3 expression was upregulated in $74 \%$ of $\mathrm{HCC}$ tumour tissues, suggesting that ANXA3 is strongly associated with the development of HCC. This result was concordant with that of other groups $(32,33)$. Western blot and quantitative PCR analyses were performed to 
Table I. Role of Annexin A3 in the development and progression of cancer.

\begin{tabular}{lll}
\hline Cancer & Expression & Role (Refs.)
\end{tabular}

$\begin{array}{ll}\text { Upper tract urothelial cancer } & \text { Upregulation in urine samples and tissues } \\ \text { Gastric cancer } & \text { Upregulation in tumour tissues }\end{array}$

Hepatocellular carcinoma Upregulation in tumour tissues

Prostate cancer

Downregulation in tumour tissues

Breast cancer

Upregulation in tumour cells

Osteosarcoma
Upregulation in osteosarcoma cell lines
Strong association with tumorigenesis

Promotes proliferation and

inhibits apoptosis of cells

Enhances the proliferation, colony

formation and migration of cells

Negatively associated with

the pT stage and Gleason score

Promotes the proliferation

and colony formation of cells

Inhibits the apoptosis of cells

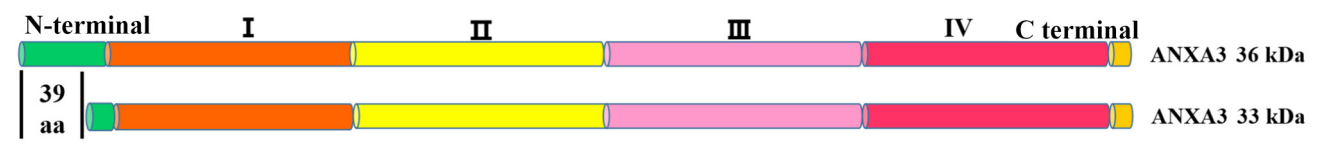

Figure 1. Diagram of the two isoforms of ANXA3. The $33 \mathrm{kDa}$ and $36 \mathrm{kDa}$ ANXA3 proteins. ANXA3 contains an N terminal, four annexin repeat domains (I-IV) and a $\mathrm{C}$ terminal. The $33 \mathrm{kDa}$ isoform of ANXA3 lacks the first 39 amino acid residues of the N-terminal region, unlike the $36 \mathrm{kDa}$ isoform. ANXA3, Annexin A3; aa, amino acids.

detect ANXA3 expression in 34 matched HCC tissues of and adjacent normal liver tissues (32). The results demonstrated that ANXA3 expression was notably higher in HCC tumour tissues compared with normal liver tissues, at both protein and mRNA levels, which was further confirmed via immunohistochemistry staining. ANXA3 expression was elevated in almost 58.1\% of 155 clinical primary HCC samples. Upregulated ANXA3 expression was positively associated with tumour size of $\mathrm{HCC}$, advanced clinical stage and number of lesions, but was negatively associated with the prognosis of HCC. Further functional investigations demonstrated that upregulated ANXA3 expression enhanced cell proliferation, colony formation, cell migration and invasion of HCC. Conversely, downregulation of ANXA3 significantly suppressed these cell behaviours. Collectively, these findings suggest that ANXA3 may severe as an independent prognostic factor for patients with HCC. ANXA3 may play an oncogenic role in promoting tumorigenesis of HCC, and thus is considered a novel target for HCC therapy (32). A previous study demonstrated that ANXA3 expression is higher in $50.6 \%$ of primary HCC clinical samples compared with matched adjacent normal liver samples (33).

The cancer stem cell (CSC), which has the capacity for tumour initiation, self-renewal and differentiation, has recently been considered to contribute to the recurrence and metastasis of HCC (34). CD133 is a molecular indicator of CSCs in HCC and is used to obtain HCC stem-like cells isolated from HCC cells (35). Pan et al reported that compared with non-CSCs, CSCs derived from Huh7 cells highly expressed endogenous ANXA3 (32). In addition, ANXA 3 plays an essential role in conferring the stemness of HCC by inhibiting the hypoxia-inducible factor (HIF)-1 $\alpha / \mathrm{NOTCH}$ pathway (34). Tong et al demonstrated that both endogenous and exogenous ANXA3 proteins can maintain the CSC characteristics of HCC cells and facilitate self-renewal and proliferation of HCC CSCs by downregulating the c-JUN
N-terminal kinase (JNK) signalling pathway (33). Recently, it has been reported that ANXA3 can promote tumorigenicity in HCC by remodelling the immune microenvironment by regulating the infiltrated neutrophil-lymphocyte ratio (iNLR) and the release of chemokines, CXCL8 and CCL25 from HCC cells (36). High ANXA3 expression is associated with high iNLR, and high risk of mortality in patients with HCC (36). Taken together, these findings suggest that ANXA3 may be a potential diagnostic marker and therapeutic target for HCC (37).

ANXA3 and the development and progression of prostate cancer. A previous study identified that ANXA3 is one of the four proteins whose expression significantly differs between 31 prostate cancer tissues and matched benign tissues on differential radioactive quantification (38). Immunohistochemistry analysis further demonstrated that ANXA3 expression was lower in the cancer group compared with the benign group, and low ANXA3 expression was dependent on the Gleason pattern of prostate cancer with a negative correlation. Köllermann et al and Jeun et al also provided powerful data regarding the association between ANXA3 and prostate cancer (39,40). These authors examined altered ANXA3 expression in prostate cancer, benign epithelium and high-grade prostatic intraepithelial neoplasia (PIN) tissues using immunohistochemistry and tissue microarray. The results demonstrated that ANXA3 expression was notably downregulated in prostate cancer tissues compared with benign epithelium and high-grade PIN tissues, and $27.2 \%$ of prostatic cancer samples negatively expressed ANXA3. Downregulated ANXA3 expression was negatively associated with the pT stage and Gleason score of prostate cancer but positively associated with poor prognosis of prostate cancer, suggesting that ANXA3 may be used as a marker for the early diagnosis of prostate cancer. Other studies have reported the value of ANXA3 in diagnosing prostate cancer $(40,41)$. 
Table II. Role of Annexin A3 in cancer resistance to drugs.

\begin{tabular}{lllr}
\hline Cancer & \multicolumn{1}{c}{ Drugs } & Resistance & (Refs.) \\
\hline Hepatocellular carcinoma & Cisplatin, 5-fluorouracil, staurosporine and sorafenib & Increase & $(33,34,45)$ \\
Ovarian carcinoma & Platinum and cisplatin & Increase & $(46-48)$ \\
Lung cancer & Platinum, cisplatin and oxaliplatin & Increase & $(49,50)$ \\
Prostate cancer & Cyclophosphamide & Increase & $(51)$ \\
Breast cancer & Doxorubicin & Increase & $(52)$ \\
Colorectal cancer & Oxaliplatin & Increase & $(53)$ \\
\hline
\end{tabular}

Western blot analysis was performed to detect ANXA3 protein expression in the urine samples of 590 patients, of whom 367 had prostate cancer and 223 had an unverified adenocarcinoma (41). The results demonstrated that the sensitivity and specificity of ANXA3 as a diagnostic marker for prostate cancer exceeded that of tPSA, since patients had negative findings on digital rectal examination and low prostate-specific antigen concentration $(2-10 \mathrm{ng} / \mathrm{ml})$. A recent study reported that ANXA3 expression in urine is clinically associated with the actual tumour volume (40). Collectively, these findings suggest that the quantification of ANXA3 expression in urine samples may be used as a specific marker for the early diagnosis of prostate cancer.

ANXA3 and the development and progression of breast cancer. ANXA3 expression is higher in breast cancer cell lines (MDA-MB 231, HCC-69 and HCC-1954) compared with other cell types (42). ANXA3 knockdown inhibits the proliferation and colony forming ability of MDA-MB 231 cells in vitro (42). In another study, the influence of ANXA3 on the incidence of breast cancer was evaluated using a nude mouse model bearing a subcutaneous tumour in vivo (43). The volume and weight of tumours in the ANXA3 silencing group was smaller compared with the control group. Furthermore, the level of red blood vessels was relatively low in the ANXA3 silencing group. The proliferation rate was lower; however, the $\mathrm{G}_{0} / \mathrm{G}_{1}$ cell ratio was higher in the transfection group compared with the control group (43). ANXA3 expression was associated with the size of the tumour and disease-free survival in 30 patients with primary breast cancer (42). Taken together, these findings suggest that ANXA3 plays an important role in the initiation and progression of breast cancer, and thus may be used as a potential therapeutic target for breast cancer.

ANXA3 and the development and progression of osteosarcoma. ANXA3 expression is higher in osteosarcoma cell lines, HOS and U2OS, compared with osteoblasts (44). To further examine the role of ANXA3 in osteosarcoma cells, ANXA3 siRNA was transfected into HOS, U2OS and osteoblasts. The mRNA and protein expression levels of ANXA3 were successfully inhibited following transfection with siRNA. The results demonstrated that ANXA3 knockdown decreased the apoptotic rate of HOS and U2OS cells by 3 -fold (44). These findings suggest that ANXA3 may regulate the tumorigenicity by influencing the apoptosis of osteosarcoma cells.
ANXA3 and the drug resistance of tumours. Recently, it has been suggested that ANXA3 may exert an important influence on the drug resistance of different types of tumours, such as HCC, ovarian carcinoma, lung adenocarcinoma (LADC) and prostatic carcinoma (Table II).

Role of ANXA3 in HCC drug resistance. To study the role of ANXA3 in drug resistance, the expression of ANXA3 was changed in different HCC cell lines by overexpressing ANXA3 or by targeting the ANXA3-specific shRNA vectors (32). Results of the in vitro drug sensitivity assay and in vivo tumour growth suppression assay indicated that overexpression of ANXA3 notably enhanced the drug resistance of HCC cells to cisplatin and 5-fluorouracil (5-FU), while ANXA3 knockdown significantly decreased the resistance to these drugs. Thus, ANXA3 may play an important role in the occurrence of drug resistance in patients with $\mathrm{HCC}$, and may serve as an innovative target for HCC treatment. Tong et al (33) reported that increased ANXA3 expression resulted in the occurrence of drug resistance among patients with HCC to cisplatin, 5-FU and staurosporine both in vitro and in vivo. Another study investigated the role of ANXA3 in the resistance of HCC cells to 5-FU (32). They established the Bel7302/5-FU cell line, which is resistant to 5-FU, and its parental Bel7302 cell line, which is sensitive to 5-FU (32). Using the quantitative proteomic method bearing isobaric labelling combined with 2D LC-MS/MS, ANXA3 expression was identified to be higher in Bel7302/5-FU compared with Be17302, which was further validated via western blot and quantitative PCR analyses. Furthermore, ANXA3 knockdown in 5-FU-resistant Bel7302/5-FU cells notably decreased the drug resistance of HCC cells to 5-FU, cisplatin and adriamycin. A recent study demonstrated that ANXA3 expression is higher in sorafenib-resistant HCC cells, HepG2 and Huh7, and sorafenib-resistant patient-derived HCC xenografts (45). Patients with low ANXA3 expression had a better prognosis after receiving sorafenib treatment than those with high ANXA3 expression. Furthermore, ANXA3 knockdown in the sorafenib-resistant HCC cells, HepG2 and Huh7, suppressed the resistance of HCC cells to sorafenib (45). Further experiments demonstrated that anti-ANXA3 therapy (anti-ANXA3 monoclonal antibody) combined with sorafenib can increase the apoptotic rate compared with sorafenib alone in sorafenib-resistant HCC cells (45). Taken together, these findings suggest that ANXA3 may be strongly associated with the multi-drug resistance (MDR) in HCC. The association between 
ANXA3 and drug resistance in HCC provides novel and important clues to further investigate MDR in other types of tumours.

Role of ANXA3 in ovarian carcinoma drug resistance. In human ovarian cancer cell lines, which are resistant to platinum, ANXA3 expression was reported to be higher than that in human epithelial ovarian cancer cell lines. ANXA3 expression notably increased in tumour tissues obtained from platinum-resistant patients with ovarian cancer than those obtained from platinum-sensitive patients (46-48). Yan et al (47) demonstrated that upregulated ANXA3 expression notably decreases the amount of cisplatin uptake and p53 expression induced by cisplatin, and ANXA3 knockdown significantly increases intracellular cisplatin concentrations in ovarian cancer cells. These results suggest that the upregulation of ANXA3 expression may be the specific mechanism responsible for the increase in ovarian cancer chemoresistance to cisplatin; however, the precise mechanism of ANXA3 leading to cisplatin resistance in ovarian cancer requires further investigation. Another study indicated that ANXA3 was detected in the ovarian cancer cell culture medium (48). The amount of secreted ANXA3 is dependent on the expression level of ANXA3 in ovarian cancer and the degree of ovarian cancer chemoresistance to cisplatin. The research demonstrated that there were more elliptical vesicles containing different concentrations of drugs in the cytoplasm of platinum-resistant ovarian cancer cell lines than in platinum-sensitive parental ovarian cancer cell lines (48). Part of the elliptical vesicles can fuse with the cell membrane, which suggests that ANXA3 expression may be owing to exocytosis and the release of exosomes. These results demonstrate that ANXA3 increases the chemoresistance of ovarian cancer to platinum by increasing the aggregation of vesicles and the exocytosis of vesicles containing drugs. Thus, ANXA3 may be a valuable target for the chemical therapy of ovarian cancer.

Role of ANXA3 in lung cancer drug resistance. A previous study investigated ANXA3 expression in lung cancer A549 cells, with and without platinum resistance, and LADC tissues (49). The results demonstrated that ANXA3 expression in platinum-resistant lung cancer A549 cells was significantly higher than that in the non-resistant groups, at both the protein and mRNA levels. In addition, ANXA3 expression was also detected in platinum-resistant LADC tissues, suggesting that ANXA3 may play an important role in the resistance of LADC to cisplatin. Platinum-resistant lung cancer A549 cells were further treated with a molecular compound that can bind with ANXA3. Analysis demonstrated that cell viability of cisplatin-resistant LADC significantly reduced, suggesting that the compound targeting ANXA3 can induce cell death in platinum-resistant lung cancer A549 cells. Thus, ANXA3 may be a novel therapeutic target to circumvent cisplatin resistance in patients with LADC (49). A recent study demonstrated that ANXA3 expression is higher in oxaliplatin (Ox)-resistant A549 lung cancer cells (A549/Ox) compared with normal A549 cells, which suggests that ANXA3 may be associated with lung cancer cell resistance to Ox (50). Further experiments demonstrated that ANXA3 knockdown in A549/Ox cells can enhance the effect of Ox on lung cancer cells (50). Collectively, these findings suggest that ANXA3 knockdown can suppress the resistance of lung cancer cells to $\mathrm{Ox}$.
Role of ANXA3 in prostate cancer drug resistance. Thoenes et al (51) established an SCID mice model, developed a prostate cancer phenotype that is resistant to metronomic cyclophosphamide (CPA) and obtained samples of prostate cancer PC3-D3 and PC4-D4 cell lines that are resistant to CPA and parental prostate cancer PC3-D3 cell lines. The results demonstrated that ANXA3 expression in prostate cancer PC3-D3 and PC4-D4 cell lines that are tolerant to CPA was higher than that in parental prostate cancer PC3-D3 cell lines. Furthermore, ANXA3 expression was notably higher in xenografts treated with CPA compared with groups without CPA treatment. These results indicate that high ANXA3 expression may enhance the drug resistance of prostate cancer to CPA, and can serve as a novel biomarker for predicting chemoresistance in prostate cancer.

Role of ANXA3 in breast cancer drug resistance. Doxorubicin (Dox) is widely used for the treatment of breast cancer. To further investigate the role of ANXA3 in the resistance of breast cancer to Dox, ANXA3 expression was inhibited in the human breast cancer cell line, MDA-MB-231 and mouse mammary cancer cell line, 4T1, and ANXA3 silencing cells were treated with Dox (52). Flow cytometric analysis demonstrated that there was increased Dox accumulation in ANXA3 silencing cells compared with normal cells (52). To further investigate the role of ANXA3 in the resistance of breast cancer to Dox in vivo, ANXA3 silencing and normal MDA-MB-231 and 4T1 cells were transplanted into the BALB/c mice. When the tumour size reached to 2-3 $\mathrm{mm}$, the mice were treated with Dox. The results demonstrated that ANXA3 silencing enhanced the sensitivity of Dox to breast cancer (52). Thus, ANXA3 may influence the resistance of breast cancer to Dox by regulating the cellular accumulation of Dox.

Role of ANXA3 in colorectal cancer drug resistance. Ox is used as a chemotherapy drug for colorectal cancer (CRC) (53). To determine the role of ANXA3 on Ox resistance in CRC, two CRC cell lines, HCT116 and SW480, were treated with Ox and exhibited resistance to Ox (HCT116/Ox and SW480/Ox) (53). The half-maximum inhibitory concentration values of Ox in HCT116/Ox and SW480/Ox cells were higher than that in their parental cells, suggesting that HCT116/Ox and SW480/Ox were resistant to Ox. Furthermore, ANXA3 expression in HCT116/Ox and SW480/Ox was higher compared with HCT116 and SW480 cells, suggesting that ANXA3 is associated with Ox resistance in CRC cells. Further experiments demonstrated that ANXA3 silencing suppressed Ox resistance in $\mathrm{CRC}$ via the mitogen-activated protein kinase signalling pathway (53). Collectively, these results suggest that ANXA3 plays an important role in CRC resistance to Ox. Thus, clinical studies are required to determine the role of ANXA3 in CRC resistance to Ox.

Role of ANXA3 in tumour metastasis. Recent studies have demonstrated that altered ANXA3 expression is closely associated with the metastasis of different types of cancer (Table III). ANXA3 expression is higher in different types of cancer tissues compared with controls, such as breast, lung, gallbladder and colon cancer tissues. Overexpression of ANXA3 promotes cancer metastasis. ANXA3 expression is downregulated in 
Table III. Role of Annexin A3 in the metastasis of cancer.

\begin{tabular}{|c|c|c|c|}
\hline Cancer & Expression & Role & (Refs.) \\
\hline Breast cancer & $\begin{array}{l}\text { Upregulation in patients with } \\
\text { lymph node metastasis }\end{array}$ & May promote metastasis & $(54-57)$ \\
\hline Lung cancer & Upregulation in patients with metastasis & May promote lymph node metastasis & $(58,59)$ \\
\hline Papillary thyroid carcinoma & Downregulation in patients with metastasis & Significantly associated with metastasis & $(60)$ \\
\hline Gallbladder carcinoma & Upregulation in patients with metastasis & May promote metastasis & $(61,62)$ \\
\hline Colon cancer & Upregulation in patients with cancer & $\begin{array}{l}\text { May promote metastasis } \\
\text { via the HIF-1 } \alpha \text { pathway }\end{array}$ & $(63-68)$ \\
\hline Pancreatic cancer & $\begin{array}{l}\text { Upregulation in patients with } \\
\text { pancreatic cancer }\end{array}$ & $\begin{array}{l}\text { Promotes epithelial-to-mesenchymal } \\
\text { transition and lymph node metastasis }\end{array}$ & $(69)$ \\
\hline
\end{tabular}

HIF, hypoxia-inducible factor.

papillary thyroid carcinoma compared with normal tissues, which promotes cancer metastasis (54-69).

Role of ANXA3 in breast cancer metastasis. Previous studies have reported that ANXA3 is expressed in several human breast cancer cell lines and 60 breast cancer tissues $(42,54)$. Western blot and immunohistochemistry analyses demonstrated that ANXA3 is highly expressed in MCF-7 and MDA-MB-435 cells, and breast cancer tissues (54). Other studies have reported the high ANXA3 expression in breast cancer cells, and ANXA3 silencing significantly suppresses the migration and invasion of breast cancer cells $(42,55)$. ANXA3 was positively expressed in $27 / 35$ patients with lymph node metastasis, and only positively expressed in 12/25 patients without lymph node metastasis (54). Further analysis demonstrated that the disease-free survival and overall survival times of patients with ANXA3-positive breast cancer were significantly shorter compared with patients with ANXA3-negative breast cancer. However, ANXA3 expression is not associated with age, hormone receptor, tumour grade and histology (54). Further studies also confirmed that ANXA3 expression is positively associated with the disease-free survival and overall survival of lymph node metastasis in patients with breast cancer $(42,56)$. ANXA3 expression is higher in patients with triple-negative breast cancer, which frequently metastasises compared with other types of cancer $(56,57)$. These results suggest that high ANXA3 expression may promote the metastasis of breast cancer. ANXA3 is an independent factor of poor prognosis in breast cancer, and thus may be a novel potential therapeutic target for predicting breast cancer metastasis $(56,57)$.

Role of ANXA3 in lung cancer metastasis. The differential protein expression between LADC tissues with and without lymph node metastasis were identified using fluorescence differential gel electrophoresis and mass spectrometry analysis (58). Western blot and immunohistochemistry analyses demonstrated that the expression of ANXA3 was higher in the metastasis group compared with the non-metastasis group. Further analysis indicated that high ANXA3 expression increased the postoperative recurrence rate and decreased the average and overall survival rate of patients with LADC, which was not associated with sex, age, tumour size and differentiation. In addition, ANXA3 silencing inhibits the invasion of lung cancer cells (59). These results suggest that ANXA3 expression may promote lymph node metastasis of lung cancer. Thus, ANXA3 may be used as a novel biomarker for predicting the metastasis and prognosis of lung cancer.

Role of ANXA3 in papillary thyroid carcinoma metastasis. Jung et al (60) analysed the differential protein expression of 25 pairs of papillary thyroid carcinomas and corresponding normal tissues by performing two-dimensional electrophoresis, mass spectrometry, western blot, northern blot and immunohistochemical analyses, and found that ANXA3 expression was lower in papillary thyroid carcinoma compared with the controls, at both mRNA and protein levels. Immunohistochemistry analysis demonstrated that ANXA3 expression in papillary thyroid carcinoma with lymph node metastasis decreased, but the differences were not significant. However, ANXA3 expression was significantly lower in papillary thyroid carcinoma with lymph node metastasis. Papillary thyroid carcinoma tissues without lymph node metastasis had a staining score of $>3$, while those with lymph node metastasis had a staining score of $\leq 3$. Further analysis revealed that ANXA3 expression was high in $61.1 \%$ of papillary microcarcinoma tissues without lymph node metastasis; however, the expression of ANXA3 was low in $67.2 \%$ of papillary thyroid carcinoma tissues with lymph node metastasis. These results suggest that low ANXA3 expression is significantly associated with the metastasis of papillary thyroid carcinoma; thus, it may be used as one of the negative predictive indexes of papillary thyroid carcinoma.

Role of ANXA3 in gallbladder carcinoma metastasis. Tan et al (61) performed two-dimensional electrophoresis and mass spectrometry to evaluate the differential protein expression of ANXA3 between six pairs of gallbladder carcinoma and benign gallbladder tissues. Immunohistochemical analysis demonstrated that high ANXA3 expression was closely associated with tumour grade, lymph node metastasis and postoperative survival of gallbladder carcinoma. Similarly, Wang et al (62) performed western blot and reverse transcription-quantitative PCR analyses, and reported that ANXA3 expression was significantly higher in GBC-SD18H cells, with high metastatic potential, compared with GBC-SD18L cells, with low metastatic potential (62). Collectively, these results suggest that ANXA3 
may promote gallbladder carcinoma metastasis. Thus, ANXA3 may be used as a therapeutic target for gallbladder carcinoma.

Role of ANXA3 in colon cancer metastasis. The mRNA levels of ANXA3 in the blood of patients with colon cancer was significantly higher compared with healthy controls $(63,64)$. Another study reported that ANXA3 expression was upregulated in colon cancer tissues, suggesting that ANXA3 may be closely associated with colon cancer (65). Xie et al analysed ANXA3 and HIF- $\alpha$ expression and their association with the clinicopathological characteristics and prognosis of 60 patients with colon cancer $(65,66)$. The results demonstrated that 65 and $47 \%$ of patients with colon cancer had high ANXA3 and HIF- $\alpha$ expression, respectively, and ANXA3 was closely associated with tumour size and Dukes' staging. HIF- $\alpha$ is as key regulator of tumour angiogenesis $(67,68)$. It can stimulate vascular endothelial growth factor (VEGF) expression in tumours $(67,68)$. The expression patterns of ANXA3 and HIF- $\alpha$ were similar, and their expression was closely associated. These results suggest that high ANXA3 expression promotes the development and metastasis of colon cancer, which may induce angiogenesis via the HIF- $\alpha$ signalling pathway.

Role of ANXA3 in pancreatic cancer metastasis. Quantitative PCR and western blot analyses demonstrated that ANXA3 was highly expressed in 115 patients with pancreatic cancer (PC) (69). ANXA3 silencing inhibits epithelial-to-mesenchymal transition (EMT) and lymph node metastasis (69). In addition, ANXA3 knockdown suppresses the migratory and invasive abilities of PC cells in vitro (69). The suppression of lymph node metastasis by ANXA3 silencing has also been confirmed in tumour xenografts in vivo (69). Taken together, these results suggest that ANXA3 silencing can inhibit the metastasis of PC. Thus, ANXA3 may be a potential therapeutic target for PC metastasis.

In conclusion, ANXA3 plays an important role in the development, metastasis and drug resistance of different types of cancer. However, the pathways by which ANXA3 exerts its role remain unclear.

\section{Pathways by which ANXA3 exerts its role}

Phosphatidylinositol-3 kinase (PI3K)/Akt signalling pathway. The PI3K/Akt signalling pathway plays an important role in cell proliferation, metabolism and migration (70). AMI is a consequence of coronary atherosclerotic heart disease, which can lead to the reduction of myocardial cells (71). An AMI rat model was constructed (71). ANXA3 shRNA was transfected into different groups of rats to inhibit ANXA3 expression. The results demonstrated that ANXA3 silencing alleviated the inflammation reaction by reducing the expression of inflammatory markers, such as interleukin (IL)-6, tumour necrosis factor $\alpha$ (TNF- $\alpha$ ) and nitric oxide (NO). Furthermore, transfection with ANXA3 shRNA increased the mRNA and protein expression levels of Bcl-2, bFGF and VEGF, and phosphorylation of Akt. These findings suggest that ANXA3 silencing may regulate cell behaviour via the PI3K/Akt signalling pathway. LY294002, an inhibitor of the PI3K/Akt signalling pathway, can activate the PI3K/Akt signalling pathway and alleviate inflammation, which is caused by ANXA3 silencing (71). In animals transfected with ANXA3 shRNA and LY294002, ANXA3 expression decreased, but the PI3K/Akt pathway was not activated (71). This suggests that ANXA3 may regulate cellular behaviour in individuals with AMI via the PI3K/Akt pathway. Thus, ANXA3 may play an important role in the development of cancer via the PI3K/Akt pathway.

$N F-\kappa B$ signalling pathway. ANXA3 expression is upregulated in breast cancer tissues compared with paracancerous tissues (52). High ANXA3 expression is associated with poor overall survival, suggesting that ANXA3 may play an important role in the development of breast cancer (52). To further evaluate its role in breast cancer, shRNA-targeted ANXA3 was transfected in vivo and vitro. The results demonstrated that ANXA3 silencing inhibited cell invasion, whilst promoting cell proliferation (52). This result remains controversial, as it is difficult to determine whether the protein is 'good' or 'bad' for the development of breast cancer. Further experiments indicated that ANXA3 knockdown inhibited the NF- $\kappa$ B pathway by increasing I $\kappa \mathrm{B} \alpha$ expression, which is a cellular protein $(52,72)$. $\mathrm{NF}-\kappa \mathrm{B}$ plays an important role in the EMT process in a breast cancer model (73). ANXA3 silencing regulates the EMT process via the $\mathrm{NF}-\kappa \mathrm{B}$ pathway. Blockade of the I $\mathrm{B} \alpha$ pathway can alleviate the EMT process induced by ANXA3 knockdown (73). These results suggest that ANXA3 may play roles in the development of other types of cancer via the NF- $\mathrm{B}$ pathway, which should be validated by further studies.

$J N K$ signalling pathway. ANXA3 is closely associated with HCC. ANXA3 expression in the sera of patients with HCC is associated with their clinical features (8). Excessive ANXA3 expression plays an important role in promoting cancer and inducing stem cell features in live CD133+ liver CSCs (33). ANXA3 exerts its role by enhancing the JNK signalling pathway, thus increasing JNK kinase activity and its expression (33). Blockade of ANXA3 with a monoclonal antibody can reduce tumour growth and self-renewal in vitro and in vivo (33). Cancer-related fibroblasts (CAFs), which are found within cancer cells, have a significant effect on the proliferation, metastasis, chemoresistance and invasion of cancer cells (74). ANXA3 expression is higher in CAFs in tumour tissues compared with normal fibroblasts (74). The CAF-conditioned medium can increase ANXA3 expression in lung cancer cells, which increases cisplatin resistance. Conversely, ANXA3 knockdown in lung cancer increases cisplatin sensitivity. ANXA3 plays an important role in the chemoresistance of lung cancer by regulating JNK activity. Using the specific JNK activity inhibitor can retard the effect of ANXA3 on cisplatin resistance. Thus, ANXA3 expression promotes HCC and lung cancer via the JNK signalling pathway (74). Another study reported that ANXA3 silencing ameliorates the intracranial aneurysm by inhibiting the JNK signalling pathway (75). Thus, ANXA3 may exert its role in different types of cancer and diseases via the JNK signalling pathway.

Extracellular signal-regulated kinase (ERK) signalling pathway. Quantitative proteomics analysis demonstrated that ANXA3 expression is higher in LADC tissues (76). Furthermore, ANXA3 knockdown inhibits the proliferation, invasion, migration and metastases of LADC cells in vivo and in vitro (76). 


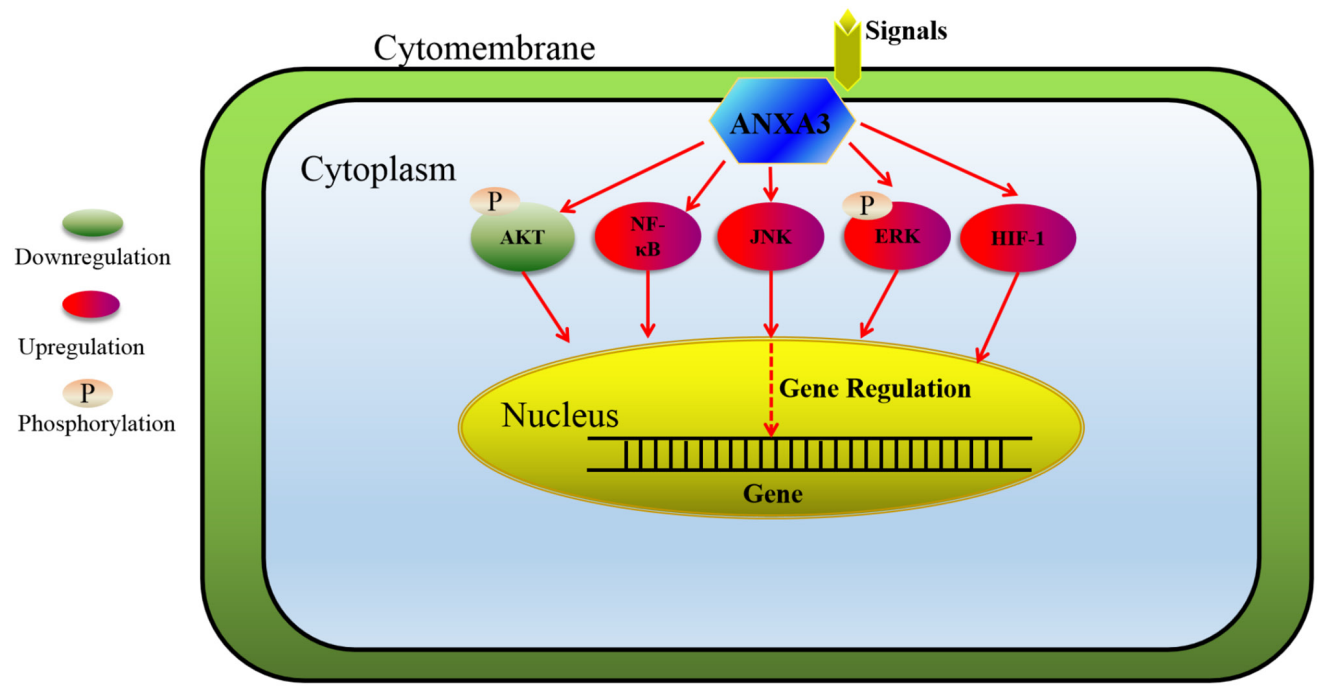

Figure 2. Signal transduction pathways by which ANXA3 exerts its role. ANXA3 exerts its role by regulating cell proliferation, migration and apoptosis by downregulating the PI3K/Akt signalling pathway and upregulating the NF- $\mathrm{BB}$, JNK, ERK and HIF-1 signalling pathways. ANXA3, Annexin A3; PI3K, phosphatidylinositol-3 kinase; NF-кB, nuclear factor-кB; JNK, c-JUN N-terminal kinase; ERK, extracellular signal-regulated kinase; HIF, hypoxia-inducible factor.

However, the molecular mechanism by which ANXA3 knockdown affects LADC cells remains unclear. It has been reported that ANXA3 silencing can reduce the phosphorylation of MEK and ERK (76). Thus, ANXA3 may exert its role via the ERK signalling pathway. In addition, ANXA3 expression is higher in two CRC cell lines, HCT116/Ox and SW480/Ox, which develop Ox resistance. ANXA3 silencing can reduce the proliferation, migration, invasion, migration and metastases of CRC cells, but promote cell apoptosis. Furthermore, ANXA3 knockdown can reduce the phosphorylation of ERK and JNK. The ERK inhibitor can reverse the changes caused by ANXA3 knockdown (53). Taken together, these findings suggest that ANXA3 may exert its role via the ERK signalling pathway in lung cancer and CRC.

HIF-1 signalling pathway. ANXA3 expression is higher in the sera of patients with melanoma compared with healthy controls (77). In addition, ANXA3 expression has a close association with the overall survival of patients with melanoma, suggesting that ANXA3 may play an important role in melanoma. Overexpression of ANXA3 can promote the proliferation and migration of human melanoma WM-115 cells. Conversely, ANXA3 knockdown inhibits cell proliferation and migration (77). Furthermore, ANXA3 exerts its role by activating HIF-1 $\alpha$ (77). Another study reported that ANXA3 increases the migration and tube formation of human umbilical vein endothelial cells (9). Furthermore, ANXA3 can induce VEGF production via the HIF-1 pathway (9). Thus, ANXA3 may play certain roles in cancer and the angiogenesis process via the HIF-1 pathway. It also plays an important role in the maintenance of cancer stem-like cells/cancer-initiating cells via the HIF pathway (78).

\section{Conclusion and prospects}

ANXA3 is closely associated with the development, drug resistance, metastasis and prognosis of several malignancies, such as prostate cancer, upper tract urothelial carcinoma, gastric cancer, hepatocellular cancer, ovarian cancer, lung cancer, breast cancer and gallbladder cancer. Its altered expression is a potential biomarker for tumour staging, grading, metastasis and prognosis of patients. Thus, it may be used as a novel target for the treatment and diagnosis of tumour. ANXA3 exerts its role via the PI3K/Akt, NF- $\kappa \mathrm{B}, \mathrm{JNK}, \mathrm{ERK}$ and HIF-1 signalling pathways (Fig. 2). In addition, ANXA3 has two isoforms ( $33 \mathrm{kDa}$ and $36 \mathrm{kDa}$ ANXA3 proteins), and the expression patterns of these two isoforms vary in different types of cells. Further studies are required to verify the function of ANXA3 and its two isoforms in tumours and their mechanisms.

\section{Acknowledgements}

Not applicable.

\section{Funding}

No funding was received.

\section{Availability of data and materials}

Not applicable.

\section{Authors' contributions}

CL and NL performed the literature review and drafted the initial manuscript. GL and XF critically revised the manuscript for important intellectual content. Data authentication is not applicable. All authors have read and approved the manuscript.

\section{Ethics approval and consent to participate}

Not applicable. 


\section{Patient consent for publication}

Not applicable.

\section{Competing interests}

The authors declare that they have no competing interests.

\section{References}

1. Lizarbe MA, Barrasa JI, Olmo N, Gavilanes F and Turnay J: Annexin-phospholipid interactions. Functional implications. Int J Mol Sci 14: 2652-2683, 2013.

2. Markoff A and Gerke V: Expression and functions of annexins in the kidney. Am J Physiol Renal Physiol 289: F949-F956, 2005.

3. Wu N, Liu S, Guo C, Hou Z and Sun MZ: The role of annexin A3 playing in cancers. Clin Transl Oncol 15: 106-110, 2013.

4. Gerke V and Moss SE: Annexins: From structure to function. Physiol Rev 82: 331-371, 2002.

5. Rescher U and Gerke V: Annexins - unique membrane binding proteins with diverse functions. J Cell Sci 117: 2631-2639, 2004

6. Ganesan T, Sinniah A, Ibrahim ZA, Chik Z and Alshawsh MA: Annexin A1: A bane or a boon in cancer? Molecules 25: 3700, 2020.

7. Christensen MV, Høgdall CK, Jochumsen KM and Høgdall EVS: Annexin A2 and cancer: A systematic review. Int J Oncol 52: 5-18, 2018.

8. Park JE, Lee DH, Lee JA, Park SG, Kim NS, Park BC and Cho S Annexin A3 is a potential angiogenic mediator. Biochem Biophys Res Commun 337: 1283-1287, 2005.

9. Mohr T, Haudek-Prinz V, Slany A, Grillari J, Micksche M and Gerner C: Proteome profiling in IL-1 $\beta$ and VEGF-activated human umbilical vein endothelial cells delineates the interlink between inflammation and angiogenesis. PLoS One 12: e0179065, 2017.

10. Niimi S, Harashima M, Gamou M, Hyuga M, Seki T, Ariga T, Kawanishi $\mathrm{T}$ and Hayakawa T: Expression of annexin A3 in primary cultured parenchymal rat hepatocytes and inhibition of DNA synthesis by suppression of annexin A3 expression using RNA interference. Biol Pharm Bull 28: 424-428, 2005.

11. Harashima M, Harada K, Ito Y, Hyuga M, Seki T, Ariga T, Yamaguchi T and Niimi S: Annexin A3 expression increases in hepatocytes and is regulated by hepatocyte growth factor in rat liver regeneration. J Biochem 143: 537-545, 2008.

12. Mussunoor S and Murray GI: The role of annexins in tumour development and progression. J Pathol 216: 131-140, 2008.

13. Huber R, Berendes R, Burger A, Luecke H and Karshikov A: Annexin V-crystal structure and its implications on function. Behring Inst Mitt 91: 107-125, 1992

14. Réty S, Sopková-de Oliveira Santos J, Dreyfuss L, Blondeau K, Hofbauerová K, Raguénès-Nicol C, Kerboeuf D, Renouard M, Russo-Marie F and Lewit-Bentley A: The crystal structure of annexin A8 is similar to that of annexin A3. J Mol Biol 345: $1131-1139,2005$.

15. Hofmann A, Raguénès-Nicol C, Favier-Perron B, Mesonero J, Huber R, Russo-Marie F and Lewit-Bentley A: The annexin A3-membrane interaction is modulated by an N-terminal tryptophan. Biochemistry 39: 7712-7721, 2000.

16. Perron B, Lewit-Bentley A, Geny B and Russo-Marie F: Can enzymatic activity, or otherwise, be inferred from structural studies of annexin III? J Biol Chem 272: 11321-11326, 1997.

17. Sopkova J,Raguenes-Nicol C,VincentM,Chevalier A,Lewit-Bentley A, Russo-Marie $\mathrm{F}$ and Gallay $\mathrm{J}$ : $\mathrm{Ca}(2+)$ and membrane binding to annexin 3 modulate the structure and dynamics of its $\mathrm{N}$ terminus and domain III. Protein Sci 11: 1613-1625, 2002.

18. Le Cabec V, Russo-Marie F and Maridonneau-Parini I: Differential expression of two forms of annexin 3 in human neutrophils and monocytes and along their differentiation. Biochem Biophys Res Commun 189: 1471-1476, 1992.

19. Nishiura H, Yamanegi K, Kawabe M, Kato-Kogoe N, Yamada N and Nakasho K: Annexin A3 plays a role in cytoplasmic calcium oscillation by extracellular calcium in the human promyelocytic leukemia HL-60 cells differentiated by phorbol-12-myristate-13-acetate. Exp Mol Pathol 97: 241-246, 2014.

20. Junker H, Suofu Y, Venz S, Sascau M, Herndon JG, Kessler C, Walther R and Popa-Wagner A: Proteomic identification of an upregulated isoform of annexin A3 in the rat brain following reversible cerebral ischemia. Glia 55: 1630-1637, 2007.
21. Hamelin-Peyron C, Vlaeminck-Guillem V,Haïdous H, Schwall GP, Poznanović S, Gorius-Gallet E, Michel S, Larue A, Guillotte M, Ruffion A, et al: Prostate cancer biomarker annexin A3 detected in urines obtained following digital rectal examination presents antigenic variability. Clin Biochem 47: 901-908, 2014.

22. Zamora DO, Riviere M, Choi D, Pan Y, Planck SR, Rosenbaum JT, David LL and Smith JR: Proteomic profiling of human retinal and choroidal endothelial cells reveals molecular heterogeneity related to tissue of origin. Mol Vis 13: 2058-2065, 2007.

23. Bianchi C, Bombelli S, Raimondo F, Torsello B, Angeloni V, Ferrero S, Di Stefano V, Chinello C, Cifola I, Invernizzi L, et al: Primary cell cultures from human renal cortex and renal-cell carcinoma evidence a differential expression of two spliced isoforms of Annexin A3. Am J Pathol 176: 1660-1670, 2010.

24. Lu CM, Lin JJ, Huang HH, Ko YC, Hsu JL, Chen JC, Din ZH and $\mathrm{Wu}$ YJ: A panel of tumor markers, calreticulin, annexin A2, and annexin A3 in upper tract urothelial carcinoma identified by proteomic and immunological analysis. BMC Cancer 14: 363, 2014.

25. Yu SY, Li Y, Fan LQ, Zhao Q, Tan BB and Liu Y: Impact of Annexin A3 expression in gastric cancer cells. Neoplasma 61: 257-264, 2014

26. Zhai JM, Sun SJ, Wang W and Zeng C: Expression of annexin A3 in gastric cancer and its correlation with proliferation and apoptosis. Asian Pac J Cancer Prev 15: 3001-3004, 2014.

27. Endl E and Gerdes J: The Ki-67 protein: Fascinating forms and an unknown function. Exp Cell Res 257: 231-237, 2000.

28. Cang S, Iragavarapu C, Savooji J, Song Y and Liu D: ABT-199 (venetoclax) and BCL-2 inhibitors in clinical development. J Hematol Oncol 8: 129, 2015 .

29. Wang $\mathrm{K}$ and $\mathrm{Li} \mathrm{J}$ : Overexpression of ANXA3 is an independent prognostic indicator in gastric cancer and its depletion suppresses cell proliferation and tumor growth. Oncotarget 7: 86972-86984, 2016.

30. Wang J, Jia X, Meng X, Li Y, Wu W, Zhang X, Xu H and Cui J: Annexin A3 may play an important role in ochratoxin-induced malignant transformation of human gastric epithelium cells. Toxicol Lett 313: 150-158, 2019.

31. Tong SW, Yang YX, Hu HD, An X, Ye F, Hu P, Ren H, Li SL and Zhang DZ: Proteomic investigation of 5-fluorouracil resistance in a human hepatocellular carcinoma cell line. J Cell Biochem 113: 1671-1680, 2012.

32. Pan QZ, Pan K, Weng DS, Zhao JJ, Zhang XF, Wang DD, Lv L, Jiang SS, Zheng HX and Xia JC: Annexin A3 promotes tumorigenesis and resistance to chemotherapy in hepatocellular carcinoma. Mol Carcinog 54: 598-607, 2015.

33. Tong M, Fung TM, Luk ST, Ng KY, Lee TK, Lin CH, Yam JW, Chan KW, Ng F, Zheng BJ, et al: ANXA3/JNK signaling promotes self-renewal and tumor growth, and its blockade provides a therapeutic target for hepatocellular carcinoma. Stem Cell Reports 5: 45-59, 2015

34. Pang RW and Poon RT: Cancer stem cell as a potential therapeutic target in hepatocellular carcinoma. Curr Cancer Drug Targets 12: 1081-1094, 2012.

35. Nguyen LV, Vanner R, Dirks P and Eaves CJ: Cancer stem cells: An evolving concept. Nat Rev Cancer 12: 133-143, 2012.

36. Zhu Q, Pan QZ, Zhong AL, Hu H, Zhao JJ, Tang Y, Hu WM, Li M, Weng DS, Chen MY, et al: Annexin A3 upregulates the infiltrated neutrophil-lymphocyte ratio to remodel the immune microenvironment in hepatocellular carcinoma. Int Immunopharmacol 89 (Pt A): 107139,2020

37. Yang XR and Guo W: ASO Author Reflections: Annexin A3 as a potential biomarker for hepatocellular carcinoma. Ann Surg Oncol 26 (Suppl 3): 529-530, 2019.

38. Wozny W, Schroer K, Schwall GP, Poznanović S, Stegmann W, Dietz K, Rogatsch H, Schaefer G, Huebl H, Klocker H, et al: Differential radioactive quantification of protein abundance ratios between benign and malignant prostate tissues: Cancer association of annexin A3. Proteomics 7: 313-322, 2007.

39. Köllermann J, Schlomm T, Bang H, Schwall GP, von Eichel-Streiber C, Simon R, Schostak M, Huland H, Berg W, Sauter G, et al: Expression and prognostic relevance of annexin A3 in prostate cancer. Eur Urol 54: 1314-1323, 2008.

40. Jeun M, Park S, Kim Y, Choi J, Song SH, Jeong IG, Kim CS and Lee KH: Self-normalized detection of ANXA3 from untreated urine of prostate cancer patients without digital rectal examination. Adv Healthc Mater 6: 1700449, 2017.

41. Schostak M, Schwall GP, Poznanović S, Groebe K, Müller M, Messinger D, Miller K, Krause H, Pelzer A, Horninger W, et al: Annexin A3 in urine: A highly specific noninvasive marker for prostate cancer early detection. J Urol 181: 343-353, 2009 . 
42. Kim JY, Jung EJ, Park HJ, Lee JH, Song EJ, Kwag SJ, Park JH, Park T, Jeong SH, Jeong CY, et al: Tumor-suppressing effect of silencing of Annexin A3 expression in breast cancer. Clin Breast Cancer 18: e713-e719, 2018

43. Li J, Zhou T, Liu L, Ju YC, Chen YT, Tan ZR and Wang J: The regulatory role of Annexin 3 in a nude mouse bearing a subcutaneous xenograft of MDA-MB-231 human breast carcinoma. Pathol Res Pract 214: 1719-1725, 2018.

44. Zeng X, Wang S, Gui P, Wu H and Li Z: Expression and significance of Annexin A3 in the osteosarcoma cell lines HOS and U2OS. Mol Med Rep 20: 2583-2590, 2019.

45. Tong M, Che N, Zhou L, Luk ST, Kau PW, Chai S, Ngan ES, Lo CM, Man K, Ding J, et al: Efficacy of annexin A3 blockade in sensitizing hepatocellular carcinoma to sorafenib and regorafenib. J Hepatol 69: 826-839, 2018.

46. Yan XD, Pan LY, Yuan Y, Lang JH and Mao N: Identification of platinum-resistance associated proteins through proteomic analysis of human ovarian cancer cells and their platinum-resistant sublines. J Proteome Res 6: 772-780, 2007.

47. Yan X, Yin J, Yao H, Mao N, Yang Y and Pan L: Increased expression of annexin A3 is a mechanism of platinum resistance in ovarian cancer. Cancer Res 70: 1616-1624, 2010.

48. Yin J, Yan X, Yao X, Zhang Y, Shan Y, Mao N, Yang Y and Pan L: Secretion of annexin A3 from ovarian cancer cells and its association with platinum resistance in ovarian cancer patients. J Cell Mol Med 16: 337-348, 2012

49. Wang C, Xiao Q, Li YW, Zhao C, Jia N, Li RL, Cao SS, Cui J, Wang $\mathrm{L}, \mathrm{Wu}$ Y, et al: Regulatory mechanisms of annexin-induced chemotherapy resistance in cisplatin resistant lung adenocarcinoma. Asian Pac J Cancer Prev 15: 3191-3194, 2014.

50. Jin YF, Huang YT and Chen PF: ANXA3 deletion inhibits the resistance of lung cancer cells to oxaliplatin. Eur Rev Med Pharmacol Sci 24: 3741-3748, 2020.

51. Thoenes L, Hoehn M, Kashirin R, Ogris M, Arnold GJ, Wagner E and Guenther M: In vivo chemoresistance of prostate cancer in metronomic cyclophosphamide therapy. J Proteomics 73: $1342-1354,2010$

52. Du R, Liu B, Zhou L, Wang D, He X, Xu X, Zhang L, Niu C and Liu S: Downregulation of annexin A3 inhibits tumor metastasis and decreases drug resistance in breast cancer. Cell Death Dis 9: 126, 2018.

53. Xu R, Yin J, Zhang Y and Zhang S: Annexin A3 depletion overcomes resistance to oxaliplatin in colorectal cancer via the MAPK signaling pathway. J Cell Biochem 120: 14585-14593, 2019

54. Zeng C, Ke Z, Song Y, Yao Y, Hu X, Zhang M, Li H and Yin J: Annexin A3 is associated with a poor prognosis in breast cancer and participates in the modulation of apoptosis in vitro by affecting the Bcl-2/Bax balance. Exp Mol Pathol 95: 23-31, 2013.

55. Zhou T, Li Y, Yang L, Liu L, Ju Y and Li C: Silencing of ANXA3 expression by RNA interference inhibits the proliferation and invasion of breast cancer cells. Oncol Rep 37: 388-398, 2017.

56. Zhou T, Li Y, Yang L, Tang T, Zhang L and Shi J: Annexin A3 as a prognostic biomarker for breast cancer: A retrospective study. BioMed Res Int 2017: 2603685, 2017

57. Zhou T, Liu S, Yang L, Ju Y and Li C: The expression of ANXA3 and its relationship with the occurrence and development of breast cancer. J BUON 23: 713-719, 2018.

58. Liu YF, Xiao ZQ, Li MX, Li MY, Zhang PF, Li C, Li F, Chen YH, Yi H, Yao HX, et al: Quantitative proteome analysis reveals annexin $\mathrm{A} 3$ as a novel biomarker in lung adenocarcinoma. J Pathol 217: 54-64, 2009.

59. Liu Q, Wang S, Pei G, Yang Y, Min X, Huang Y and Liu J: Impact analysis of miR-1253 on lung cancer progression through targeted regulation of ANXA3. Cancer Manag Res 13: 1767-1776, 2021.

60. Jung EJ, Moon HG, Park ST, Cho BI, Lee SM, Jeong CY, Ju YT, Jeong SH, Lee YJ, Choi SK, et al: Decreased annexin A3 expression correlates with tumor progression in papillary thyroid cancer. Proteomics Clin Appl 4: 528-537, 2010.

61. Tan Y, Meng HP, Wu Q, Wang FQ and Wu HR: Proteomic study of gallbladder cancer, with special reference on the expression and significance of annexin A3. Zhonghua Bing Li Xue Za Zhi 39: 382-386, 2010 (In Chinese)
62. Wang JW, Peng SY, Li JT, Wang Y, Zhang ZP, Cheng Y, Cheng DQ, Weng WH, Wu XS, Fei XZ, et al: Identification of metastasis-associated proteins involved in gallbladder carcinoma metastasis by proteomic analysis and functional exploration of chloride intracellular channel 1. Cancer Lett 281: 71-81, 2009.

63. Yip KT, Das PK, Suria D, Lim CR, Ng GH and Liew CC: A case-controlled validation study of a blood-based seven-gene biomarker panel for colorectal cancer in Malaysia. J Exp Clin Cancer Res 29: 128, 2010

64. Marshall KW, Mohr S, Khettabi FE, Nossova N, Chao S, Bao W, Ma J, Li XJ and Liew CC: A blood-based biomarker panel for stratifying current risk for colorectal cancer. Int J Cancer 126: 1177-1186, 2010

65. Madoz-Gúrpide J, López-Serra P, Martínez-Torrecuadrada JL, Sánchez L, Lombardía L and Casal JI: Proteomics-based validation of genomic data: Applications in colorectal cancer diagnosis. Mol Cell Proteomics 5: 1471-1483, 2006

66. Xie YQ, Fu D, He ZH and Tan QD: Prognostic value of Annexin A3 in human colorectal cancer and its correlation with hypoxia-inducible factor-1 $\alpha$. Oncol Lett 6: 1631-1635, 2013.

67. Katsuta M,Miyashita M, Makino H,Nomura T, Shinji S, Yamashita K, Tajiri T, Kudo M, Ishiwata T and Naito Z: Correlation of hypoxia inducible factor-1alpha with lymphatic metastasis via vascular endothelial growth factor-C in human esophageal cancer. Exp Mol Pathol 78: 123-130, 2005.

68. Kimura S, Kitadai Y, Tanaka S, Kuwai T, Hihara J, Yoshida K, Toge $\mathrm{T}$ and Chayama K: Expression of hypoxia-inducible factor (HIF)-1alpha is associated with vascular endothelial growth factor expression and tumour angiogenesis in human oesophageal squamous cell carcinoma. Eur J Cancer 40: 1904-1912, 2004.

69. Wan X, Guo D, Zhu Q and Qu R: microRNA-382 suppresses the progression of pancreatic cancer through the PI3K/Akt signaling pathway by inhibition of Anxa3. Am J Physiol Gastrointest Liver Physiol 319: G309-G322, 2020.

70. Pompura SL and Dominguez-Villar M: The PI3K/AKT signaling pathway in regulatory T-cell development, stability, and function. J Leukoc Biol 103: 1065-1076, 2018.

71. Meng H, Zhang Y, An ST and Chen Y: Annexin A3 gene silencing promotes myocardial cell repair through activation of the PI3K/Akt signaling pathway in rats with acute myocardial infarction. J Cell Physiol 234: 10535-10546, 2019.

72. Jacobs MD and Harrison SC: Structure of an IkappaBalpha/ NF-kappaB complex. Cell 95: 749-758, 1998.

73. Huber MA, Azoitei N, Baumann B, Grünert S, Sommer A, Pehamberger H, Kraut N, Beug H and Wirth T: NF-kappaB is essential for epithelial-mesenchymal transition and metastasis in a model of breast cancer progression. J Clin Invest 114: 569-581, 2004.

74. Wang L, Li X, Ren Y, Geng H, Zhang Q, Cao L, Meng Z, Wu X, Xu $\mathrm{M}$ and $\mathrm{Xu} \mathrm{K}$ : Cancer-associated fibroblasts contribute to cisplatin resistance by modulating ANXA3 in lung cancer cells. Cancer Sci 110: 1609-1620, 2019.

75. Wang Y, Wang C, Yang Q and Cheng YL: ANXA3 silencing ameliorates intracranial aneurysm via inhibition of the JNK signaling pathway. Mol Ther Nucleic Acids 17: 540-550, 2019.

76. Liu YF, Liu QQ, Zhang YH and Qiu JH: Annexin A3 knockdown suppresses lung adenocarcinoma. Anal Cell Pathol (Amst) 2016: $4131403,2016$.

77. Xu B, Zhang X, Gao Y, Song J and Shi B: Microglial Annexin A3 promoted the development of melanoma via activation of hypoxia-inducible factor-1 $\alpha$ /vascular endothelial growth factor signaling pathway. J Clin Lab Anal 35: e23622, 2021.

78. Pan QZ, Pan K, Wang QJ, Weng DS, Zhao JJ, Zheng HX, Zhang XF, Jiang SS, Lv L, Tang Y, et al: Annexin A3 as a potential target for immunotherapy of liver cancer stem-like cells. Stem Cells 33: 354-366, 2015.

This work is licensed under a Creative Commons Attribution-NonCommercial-NoDerivatives 4.0 International (CC BY-NC-ND 4.0) License. 